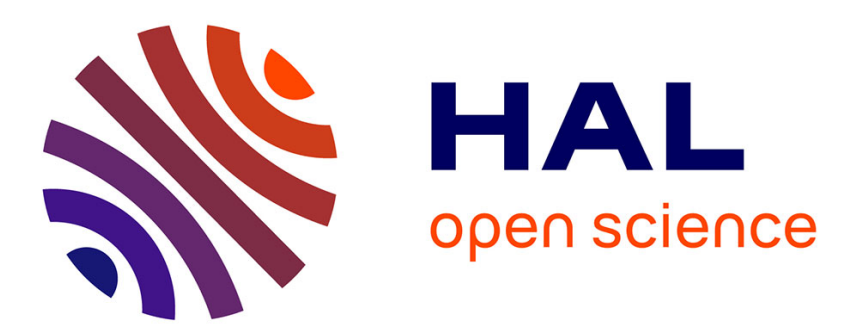

\title{
A note on the steady state optimization of the biogas production in a two-stage anaerobic digestion model Terence Bayen, Pedro Gajardo
}

\section{To cite this version:}

Terence Bayen, Pedro Gajardo. A note on the steady state optimization of the biogas production in a two-stage anaerobic digestion model. Journal of Mathematical Biology, 2019, 78 (4), pp.1067-1087. 10.1007/s00285-018-1301-3 . hal-01586942

\section{HAL Id: hal-01586942 \\ https://hal.science/hal-01586942}

Submitted on 13 Sep 2017

HAL is a multi-disciplinary open access archive for the deposit and dissemination of scientific research documents, whether they are published or not. The documents may come from teaching and research institutions in France or abroad, or from public or private research centers.
L'archive ouverte pluridisciplinaire HAL, est destinée au dépôt et à la diffusion de documents scientifiques de niveau recherche, publiés ou non, émanant des établissements d'enseignement et de recherche français ou étrangers, des laboratoires publics ou privés. 


\title{
A note on the steady state optimization of the biogas production in a two-stage anaerobic digestion model
}

\author{
Terence Bayen*†, Pedro Gajardo ${ }^{\ddagger}$
}

September 12, 2017

\begin{abstract}
In this paper, we study the optimization problem of maximizing biogas production at the steady state in a two-stage anaerobic digestion model, which was initially proposed in [4]. Nominal operating points, consisting in steady states where the involved microorganisms coexist, are usually referred to as desired operational conditions, in particular for maximizing biogas production. Nevertheless, we prove that under some conditions related to input substrate concentrations and microorganism growth functions, the optimal steady state can be the extinction of one of the two species. We provide some numerical examples of this situation.
\end{abstract}

Keywords. Anaerobic digestion, Biotechnology, Steady state analysis, Equilibrium, Optimization.

\section{Introduction}

Within the context of renewable energies, anaerobic digestion is now an attractive alternative to carbon fossil, being a well-known and established technology to treat waste in the methanization of sewage sludge from wastewater treatment plants [8]. Anaerobic digestion is a complex process that can take place in one or several bioreactors used for the production of biogas (methane, hydrogen); one of the most important drawbacks, however, is its sensitivity to disturbances, which can lead to instability problems in addition to diminishing biogas production flow [9]. For this reason, mechanistic mathematical models are a good basis for monitoring and developing control strategies to optimize the functioning of this type of process (a complete review about modeling and identification of anaerobic digestion processes can be found in [6]).

In the last thirty years, complex models of anaerobic digestion have been developed and fitted with experimental data to predict, for example, biogas production rates. This is true in the case of the ADM1 [1], a model that consists of 29 dynamic state variables, which makes it very difficult to carry out qualitative analysis. Simpler models focusing on different subprocesses of ADM1 have been proposed, as in [4] (two stages considered: acidogenesis and methanogenesis), [15] (three stages considered: acidogenesis, acetogenesis, and methanogenesis), and [5] (four stages considered: hydrolysis, acidogenesis, acetogenesis, and methanogenesis). Mathematical analysis was carried out for these models in $[11,12,13,3,15,5]$. Concerning the two-stage model proposed in [4], in [3], the authors present a complete analysis developed in a generic way that is related to the existence of steady states and their stability properties. The problem of optimizing biogas production for the model proposed in [4] is studied in [11, 12, 13]. This issue is also analyzed in [15] and [5], where models with more stages for the anaerobic digestion process are considered. In these works, one observes that steady states can be characterized as follows: (i) the extinction (washout) of one or more of the involved microorganisms and (ii) the coexistence of microorganisms. The coexistence steady states are referred to as nominal operating points, and they are typically viewed as the desired operating conditions.

\footnotetext{
*Institut Montpelliérain Alexander Grothendieck, CNRS, Univ. Montpellier. terence.bayen@umontpellier.fr

${ }^{\dagger}$ UMR INRA-SupAgro 729 'MISTEA' 2 place Viala 34060 Montpellier.

‡Departamento de Matemática, Universidad Técnica Federico Santa María, Avenida España 1680, Valparaíso, Chile pedro.gajardo@usm.cl
} 
From a practical point of view, an interesting issue is to obtain an efficient management policy for anaerobic digestion processes in order to maximize biogas production over a given period (see e.g. [7]). This policy can be characterized in a two-step optimization procedure. First, one finds an equilibrium point maximizing biogas production at the steady state, and then, one determines a control strategy in order to reach this steady state, eventually optimizing another criterion (e.g.: time, as in [2], and cost as in [11], etc.). Intuitively, the optimal steady states should be nominal operating points (coexistence of microorganims), as assumed in $[11,12]$ for the two-stage model introduced in [4]. In [15], for a three-stage model, the authors show numerically that, surprisingly, the optimal biogas production does not always occur at a steady state where all microorganisms coexist, the same phenomena that can be observed numerically in [5] for a four-stage model.

In the present work, we consider the two-stage model introduced in [4], and we prove that, under some (simple) assumptions related to input substrate concentrations and microorganism growth functions, the optimal steady state can involve the extinction of one of the species. The mathematical proof of this non-intuitive solution can be interesting from a practical point of view if the goal is to maximize biogas production. From a mathematical point of view, the problem amounts to the maximization of the supremum of two concave functions, and it is addressed by utilizing standard tools of analysis.

The paper is organized as follows: In the next section, we present the two-stage anaerobic digestion model introduced in [4], establishing a general hypotheses for the analysis and proposing an equivalent model (with the same structure) in order to reduce, for convenience, the quantity of parameters. In the third section, we recall the steady state analysis carried out in [3], which will be useful to compute the equilibrium points of the dynamics. In the fourth section, we study the optimization problem of maximizing biogas production at the steady state: Proposition 4.4 is our main result and summarizes which steady state is optimal depending on the input substrate concentrations. Finally, in the last two sections, we present some numerical examples highlighting the previous results (Section 5) and final conclusions (Section 6).

\section{Two-stage anaerobic digestion model}

A representation of the two-stage anaerobic digestion model is based on the coupling of two main reactions called acidogenesis and methanization. These two reactions can be described by the so-called AM2 model (see [4]) represented by the following dynamical system:

$$
\left\{\begin{aligned}
\dot{X}_{1} & =\left(\tilde{\mu}_{1}\left(S_{1}\right)-\alpha D\right) X_{1} \\
\dot{S}_{1} & =-k_{1} \tilde{\mu}_{1}\left(S_{1}\right) X_{1}+D\left(S_{i n}^{1}-S_{1}\right) \\
\dot{X}_{2} & =\left(\tilde{\mu}_{2}\left(S_{2}\right)-\alpha D\right) X_{2} \\
\dot{S}_{2} & =k_{2} \tilde{\mu}_{1}\left(S_{1}\right) X_{1}-k_{3} \tilde{\mu}_{2}\left(S_{2}\right) X_{2}+D\left(S_{i n}^{2}-S_{2}\right)
\end{aligned}\right.
$$

that is based on the chemostat model (see [10, 14]). Here, $X_{i}, i=1,2$, denotes the biomass concentration, $S_{1}$ is the concentration of the organic substrate characterized by its COD (Chemical Oxygen Demand) and measured in [g/l], and $S_{2}$ is the total concentration of VFA (Volatile Fatty Acids) measured in [mmol/l]. The dilution rate of the continuously operated bioreactor is denoted by $D$ (i.e., $D=Q / V$ where $Q$ is the input and output flow rate of water and $V$ is the constant volume of water present in the bioreactor). The index $i=1$, resp. $i=2$, is for the acidogenesis reaction, resp. methanization reaction. The parameters $S_{i n}^{i}$ represent the input substrate concentrations. Coefficients $k_{j}, j=1,2,3$ are positive parameters called pseudostochiometric coefficients associated to the bioreactions, $\alpha \in(0,1]$ is a parameter making it possible to decouple the HRT (Hydraulic Retention Time) and the SRT (Solid Retention Time) (see [4,3]), and the functions $\tilde{\mu}_{i}$ are the so-called growth rate functions. Values (borrowed from [4]) and units of all involved parameters are specified in Table 2 of Section 5.

The dilution rate $D$ is the decision variable due to the fact that one can control the input and output flow rates, both being equal in a continuously operated bioreactor.

The growth functions or kinetics $\tilde{\mu}_{1}(\cdot)$ and $\tilde{\mu}_{2}(\cdot)$ are usually of a Monod and Haldane type (see Example 2.1 for the definitions), respectively, as in $[4,11,12]$. However, in this paper, we consider generic kinetics $\tilde{\mu}_{1}(\cdot)$ and $\tilde{\mu}_{2}(\cdot)$, satisfying the following qualitative properties: 
Assumption 2.1. The function $\tilde{\mu}_{1}:[0,+\infty) \longrightarrow \mathbb{R}$ is concave, increasing, and continuously differentiable, with $\tilde{\mu}_{1}(0)=0$ and $\lim _{S_{1} \rightarrow+\infty} \bar{\mu}_{1}\left(S_{1}\right)=\bar{\mu}_{1}$, for some $\bar{\mu}_{1}>0$.

Assumption 2.2. The function $\tilde{\mu}_{2}:[0,+\infty) \longrightarrow \mathbb{R}$ is continuously differentiable, and for some $S_{2}^{\text {max }}>0$, it is concave and increasing over $\left[0, S_{2}^{\max }\right]$ and decreasing over $\left(S_{2}^{\max },+\infty\right)$, with $\tilde{\mu}_{2}(0)=0$ and $\tilde{\mu}_{2}\left(S_{2}\right)>0$ for all $S_{2}>0$.

Remark 2.1. Observe that with the previous assumptions, one has:

- $\tilde{\mu}_{1}^{\prime}\left(S_{1}\right)>0$ for all $S_{1}>0$, where $\tilde{\mu}_{1}^{\prime}(\cdot)$ is the derivative of function $\tilde{\mu}_{1}(\cdot)$;

- The function $\tilde{\mu}_{1}^{\prime}(\cdot)$ is decreasing over $(0,+\infty)$;

- $\tilde{\mu}_{2}^{\prime}\left(S_{2}\right)>0$ for all $S_{2} \in\left(0, S_{2}^{\max }\right), \tilde{\mu}_{2}^{\prime}\left(S_{2}^{\max }\right)=0$, and $\tilde{\mu}_{2}^{\prime}\left(S_{2}\right)<0$ for all $S_{2} \in\left(S_{2}^{\max },+\infty\right)$. Therefore, $\tilde{\mu}_{2}(\cdot)$ attains its maximum value at $S_{2}=S_{2}^{\max }$;

- The function $\tilde{\mu}_{2}^{\prime}(\cdot)$ is decreasing over $\left(0, S_{2}^{\max }\right]$.

Example 2.1. It is straightforward to check that if $\tilde{\mu}_{1}(\cdot)$ is a Monod function and $\tilde{\mu}_{2}(\cdot)$ is of Haldane type, they satisfy assumptions 2.1 and 2.2. Recall that the Monod function is defined by

$$
\tilde{\mu}_{1}\left(S_{1}\right)=\frac{\bar{\mu}_{1} S_{1}}{k_{A}+S_{1}}
$$

and the Haldane function by

$$
\tilde{\mu}_{2}\left(S_{2}\right)=\frac{\bar{\mu}_{2} S_{2}}{k_{M}+S_{2}+S_{2}^{2} / k_{I}}
$$

where $\bar{\mu}_{1}, k_{A}, \bar{\mu}_{2}, k_{M}$, and $k_{I}$ are positive parameters. In this case, the value $S_{2}^{\text {max }}$ in Assumption 2.2 is given by $S_{2}^{\max }=\sqrt{k_{M} k_{I}}$.

Finally, we introduce the expression of the methane flow rate (see [4]), given by

$$
q_{M}\left(X_{2}, S_{2}\right)=\bar{q} \tilde{\mu}_{2}\left(S_{2}\right) X_{2},
$$

where $\bar{q}>0$. The objective of the paper is to find a stable (at least locally) equilibrium point of (2.1) for which this quantity at steady state is maximal.

\section{$2.1 \quad$ Reduction of the model}

It is straightforward to verify that the AM2 model can be equivalently written with adimensioned pseudostochiometric coefficients as follows:

$$
\left\{\begin{array}{l}
\dot{x}_{1}=\mu_{1}\left(s_{1}\right) x_{1}-u x_{1} \\
\dot{s}_{1}=-\mu_{1}\left(s_{1}\right) x_{1}+\frac{u}{\alpha}\left(s_{i n}^{1}-s_{1}\right) \\
\dot{x}_{2}=\mu_{2}\left(s_{2}\right) x_{2}-u x_{2} \\
\dot{s}_{2}=-\mu_{2}\left(s_{2}\right) x_{2}+\mu_{1}\left(s_{1}\right) x_{1}+\frac{u}{\alpha}\left(s_{i n}^{2}-s_{2}\right)
\end{array}\right.
$$

From $(2.1)$, the change of variables and the new definitions of growth functions $\mu_{1}(\cdot)$ and $\mu_{2}(\cdot)$, for obtaining (2.3) are indicated in Table 1.

Remark 2.2. Note that functions $\mu_{1}(\cdot)$ and $\mu_{2}(\cdot)$, in system $(2.3)$ have exactly the same properties of $\tilde{\mu}_{1}(\cdot)$ and $\tilde{\mu}_{2}(\cdot)$, introduced in assumptions 2.1 and 2.2. The value $s_{2}^{\max }$ where $\mu_{2}(\cdot)$ is maximized is $s_{2}^{\max }=\frac{k_{1}}{k_{2}} S_{2}^{\max }$, where $S_{2}^{\max }$ is given in Assumption 2.2.

The expression (2.2) of the methane flow rate in the new variables then becomes

$$
q_{M}\left(x_{2}, s_{2}\right)=\frac{\bar{q} k_{2}}{k_{1} k_{3}} \mu_{2}\left(s_{2}\right) x_{2}
$$




\begin{tabular}{|c|c|}
\hline New variables in system $(2.3)$ & Original variables in system $(2.1)$ \\
\hline$x_{1}$ & $k_{1} X_{1}$ \\
\hline$s_{1}$ & $S_{1}$ \\
\hline$x_{2}$ & $\frac{k_{1} k_{3}}{k_{2}} X_{2}$ \\
\hline$s_{2}$ & $\frac{k_{1}}{k_{2}} S_{2}$ \\
\hline$u$ & $\alpha D$ \\
\hline$s_{i n}^{1}$ & $S_{i n}^{1}$ \\
\hline$s_{i n}^{2}$ & $\frac{k_{1}}{k_{2}} S_{i n}^{2}$ \\
\hline$\mu_{1}\left(s_{1}\right)$ & $\tilde{\mu}_{1}\left(s_{1}\right)=\tilde{\mu}_{1}\left(S_{1}\right)$ \\
\hline$\mu_{2}\left(s_{2}\right)$ & $\tilde{\mu}_{2}\left(\frac{k_{2}}{k_{1}} s_{2}\right)=\tilde{\mu}_{2}\left(S_{2}\right)$ \\
\hline
\end{tabular}

Table 1: Change of variables for obtaining (2.3) from (2.1).

\section{$3 \quad$ Steady states and stability results}

Following $[11,12,13]$, it can be interesting to suppose the dynamical system (2.3) (equivalent to (2.1)) at the steady state for a constant dilution rates $u \geq 0$ maximizing the static production of methane (2.4). Then, the bioreactor can be stabilized at the corresponding equilibrium, which then guarantees a certain value of the methane production over time. For this purpose, when $u \geq 0$ is constant in (2.3), we shall recall possible steady states admitted (i.e. with non negative values) by this system, and the stability results associated to each of them. A rigorous mathematical analysis can be found in [3].

For a fixed $u \geq 0$, we denote by $\tilde{E}^{*}(u):=\left(\bar{x}_{1}(u), \bar{s}_{1}(u), \bar{x}_{2}(u), \bar{s}_{2}(u)\right)$ a steady state of $(2.3)$. It is straightforward to check that

$$
\bar{x}_{1}(u)=\frac{\left(s_{i n}^{1}-\bar{s}_{1}(u)\right)}{\alpha} \quad \text { and } \quad \bar{x}_{2}(u)=\frac{\left(s_{i n}^{1}+s_{i n}^{2}-\bar{s}_{1}(u)-\bar{s}_{2}(u)\right)}{\alpha} .
$$

Therefore, the steady states are characterized by the values $\left(\bar{s}_{1}(u), \bar{s}_{2}(u)\right)$ only, and for this reason, we shall only write $E^{*}(u):=\left(\bar{s}_{1}(u), \bar{s}_{2}(u)\right)$ instead of $\tilde{E}^{*}(u)$.

In [3], the authors show that under Assumptions 2.1 and 2.2, there exist at least four steady states:

- $E_{1}^{*}(u)=\left(s_{i n}^{1}, s_{i n}^{2}\right)$ (total washout): This steady state always exists, and for $u$ large enough, it is globally asymptotically stable;

- $E_{2}^{*}(u)=\left(s_{i n}^{1}, s_{2}^{*}(u)\right)$ (washout of the acidogenic microorganism), where $s_{2}^{*}(u)$ is the unique solution in $\left[0, s_{2}^{\max }\right]$ of the equation $\mu_{2}\left(s_{2}\right)=u$ : It exists if $u<\max _{s \in\left[0, s_{i n}^{2}\right]} \mu_{2}(s)$. If only $E_{1}^{*}(u)$ and $E_{2}^{*}(u)$ exist, then $E_{2}^{*}(u)$ is globally asymptotically stable. Otherwise, it is unstable;

- $E_{3}^{*}(u)=\left(s_{1}^{*}(u), s_{i n}^{1}+s_{i n}^{2}-s_{1}^{*}(u)\right)$ (washout of the methanogenic microorganism), where $s_{1}^{*}(u)$ is the unique solution in $\left[0, s_{i n}^{1}\right]$ of the equation $\mu_{1}\left(s_{1}\right)=u$ : It exists if $u<\mu_{1}\left(s_{i n}^{1}\right)$. If only $E_{1}^{*}(u)$ and $E_{3}^{*}(u)$ exist, then $E_{3}^{*}(u)$ is globally asymptotically stable. Otherwise, it is unstable;

- $E_{4}^{*}(u)=\left(s_{1}^{*}(u), s_{2}^{*}(u)\right)$ (coexistence): It exists if $u<\mu_{1}\left(s_{i n}^{1}\right)$ and $u<\underset{s \in\left[0, s_{i n}^{1}+s_{i n}^{2}-s_{1}^{*}(u)\right]}{\max } \mu_{2}(s)$. In this case, it can be globally asymptotically stable or just locally stable.

If in addition to Assumption 2.2 one supposes $\lim _{s_{2} \rightarrow+\infty} \mu_{2}\left(s_{2}\right)=0$, it is shown in [3] that there could exist two other steady states:

- $\bar{E}_{2}^{*}(u)=\left(s_{i n}^{1}, s_{2}^{* *}(u)\right)$ (washout of the methanogenic microorganism), where $s_{2}^{* *}(u)$ is the unique solution in $\left[s_{2}^{\max },+\infty\right)$ of the equation $\mu_{2}\left(s_{2}\right)=u$, and 
- $\bar{E}_{4}^{*}(u)=\left(s_{1}^{*}(u), s_{2}^{* *}(u)\right)$ (coexistence).

Steady states $\bar{E}_{2}^{*}(u)$ and $\bar{E}_{4}^{*}(u)$ exist under the same conditions as $E_{2}^{*}(u)$ and $E_{4}^{*}(u)$, respectively. We do not consider these equilibrium points because they are unstable, and since our aim is to maximize the methane flow at equilibrium, this quantity evaluated in $\bar{E}_{2}^{*}(u)$ and $\bar{E}_{4}^{*}(u)$ is strictly lower than $(2.4)$ evaluated at $E_{2}^{*}(u)$ and $E_{4}^{*}(u)$, respectively. Indeed, from the respective values of $\bar{x}_{1}(u)$ and $\bar{x}_{2}(u)$ in $(3.1)$, we deduce that $\bar{x}_{2}(u)$ associated to $s_{2}^{*}(u)$ is strictly greater than $\bar{x}_{2}(u)$ associated to $s_{2}^{* *}(u)$. Since $\mu_{2}\left(s_{2}^{*}(u)\right)=\mu_{2}\left(s_{2}^{* *}(u)\right)=u$, from (2.4) we obtain that the methane flow rate evaluated at unstable equilibria $\bar{E}_{2}^{*}(u)$ and $\bar{E}_{4}^{*}(u)$ are lower.

\section{Steady state optimization}

In this section, our aim is to optimize the methane flow rate given by (2.4) at the steady state operation. First, note that this quantity is zero at the equilibria $E_{1}^{*}(u)$ and $E_{3}^{*}(u)$ introduced in the previous section (because from (3.1) one has $\left.\bar{x}_{2}(u)=0\right)$. Therefore, the objective is to find a dilution rate $u \geq 0$ such that the biogas flow rate, evaluated in $E_{2}^{*}(u)$ or $E_{4}^{*}(u)$, is maximal.

Remark 4.1. Observe that if $E_{2}^{*}(u)$ and $E_{4}^{*}(u)$ exist (for this situation, it is necessary that $u<\mu_{1}\left(s_{\text {in }}^{1}\right)$ ), the methane flow rate evaluated in $E_{2}^{*}(u)$ is lower than this quantity evaluated in $E_{4}^{*}(u){ }^{1}$ Thus, if the maximal biogas flow rate is obtained for $u<\mu_{1}\left(s_{i n}^{1}\right)$, we know that this quantity is attained at the steady state $E_{4}^{*}(u)$. Therefore, in order to maximize the methane flow rate, we only have to consider $E_{2}^{*}(u)$ or $E_{4}^{*}(u)$ in the following cases:

- Consider only $E_{4}^{*}(u)$ if $u<\mu_{1}\left(s_{i n}^{1}\right)$ and $u<\max _{s \in\left[0, s_{i n}^{1}+s_{i n}^{2}-s_{1}^{*}(u)\right]} \mu_{2}(s)$;

- Consider only $E_{2}^{*}(u)$ if $u \geq \mu_{1}\left(s_{i n}^{1}\right)$ and $u<\max _{s \in\left[0, s_{i n}^{2}\right]} \mu_{2}(s)$.

Note that the above conditions on the decision variable $u$ can be written in the form $u \in I(u):=\left[0, \mu_{2}\left(s_{2}^{+}(u)\right)\right]$, where

$$
s_{2}^{+}(u):=\underset{s \in[0, \lambda(u)]}{\operatorname{Argmax}} \mu_{2}(s)=\min \left\{\lambda(u), s_{2}^{\max }\right\},
$$

with

$$
\lambda(u):=s_{i n}^{1}+s_{i n}^{2}-\tilde{s}_{1}^{*}(u),
$$

and

$$
\tilde{s}_{1}^{*}(u):= \begin{cases}s_{1}^{*}(u)=\mu_{1}^{-1}(u) & \text { if } u<\mu_{1}\left(s_{i n}^{1}\right), \\ s_{i n}^{1} & \text { if } u \geq \mu_{1}\left(s_{i n}^{1}\right) .\end{cases}
$$

Using the definition of $\tilde{s}_{1}^{*}(u)$ given in (4.3), for a fixed $u$, we can write in a unified expression the maximal methane flow rate possible to obtain among the existing steady states (for this decision variable $u$ ), that is, the methane flow rate $(2.4)$ evaluated in $E_{2}^{*}(u)$ (when $E_{4}^{*}(u)$ does not exist) or evaluated in $E_{4}^{*}(u)$ (when $E_{2}^{*}(u)$ and $E_{4}^{*}(u)$ exist). This expression is:

$$
q_{M}(u)=\frac{\bar{q} k_{2}}{k_{1} k_{3}} \mu_{2}\left(s_{2}^{*}(u)\right)\left(s_{i n}^{1}+s_{i n}^{2}-\tilde{s}_{1}^{*}(u)-s_{2}^{*}(u)\right)=\frac{\bar{q} k_{2}}{k_{1} k_{3}} u\left(s_{i n}^{1}+s_{i n}^{2}-\tilde{s}_{1}^{*}(u)-s_{2}^{*}(u)\right) .
$$

Therefore, maximizing the methane flow rate is equivalent to solving the following optimization problem

$$
\max _{u \in I(u)} g(u)
$$

where $g:[0,+\infty) \longrightarrow[0,+\infty)$ is defined by

$$
g(u):=\frac{k_{1} k_{3}}{\bar{q} k_{2}} q_{M}(u)=\mu_{2}\left(s_{2}^{*}(u)\right)\left(s_{i n}^{1}+s_{i n}^{2}-\tilde{s}_{1}^{*}(u)-s_{2}^{*}(u)\right)=u\left(s_{i n}^{1}+s_{i n}^{2}-\tilde{s}_{1}^{*}(u)-s_{2}^{*}(u)\right),
$$

\footnotetext{
${ }^{1}$ Perhaps because of this fact some authors consider the coexistence (represented by $E_{4}^{*}(u)$ ) as a desired operating point. Nevertheless, it could happen that the methane flow rate at $E_{2}^{*}(u)$ (for some $u$ where $E_{4}^{*}(u)$ does not exist) could be greater than this flow rate evaluated in any $E_{4}^{*}(u)$ (for $u$ such that $E_{4}^{*}(u)$ exists).
} 
with $\tilde{s}_{1}^{*}(u)$ defined by $(4.3)$ and $s_{2}^{*}(u)$ is the unique solution in $\left[0, s_{2}^{\max }\right]$ of the equation $\mu_{2}\left(s_{2}\right)=u$, which exists when $u \in I(u)$.

The condition $u \in I(u)$ (necessary and sufficient for the existence of $s_{2}^{*}(u)$ ) can be written in a simpler way. In order to study this point, let us first prove some properties of the function $\phi: \mathbb{R}_{+} \longrightarrow \mathbb{R}_{+}$defined by

$$
\phi(u):=\mu_{2}\left(s_{2}^{+}(u)\right),
$$

where $s_{2}^{+}(u)$ is defined in (4.1). The function $\phi$ is depicted in Figure 1.

Lemma 4.1. Defining

$$
\tilde{s}_{2}:=\min \left\{s_{2}^{\max }, s_{i n}^{2}\right\} \quad \text { and } \quad \hat{s}_{2}:=\min \left\{s_{2}^{\max }, s_{i n}^{1}+s_{i n}^{2}\right\},
$$

the function $\phi: \mathbb{R}_{+} \longrightarrow \mathbb{R}_{+}$has the following properties:

(a) $\phi(u)=\mu_{2}\left(\tilde{s}_{2}\right)$ for all $u \geq \mu_{1}\left(s_{i n}^{1}\right)$;

(b) $\phi$ is non-increasing. In particular, if $\tilde{s}_{2}=s_{2}^{\max }$, then $\phi(u)=\mu_{2}\left(\tilde{s}_{2}\right)$ for all $u \geq 0$;

(c) $\phi(0)=\mu_{2}\left(\hat{s}_{2}\right) \geq \mu_{2}\left(\tilde{s}_{2}\right)$.

Proof. For $u \geq \mu_{1}\left(s_{i n}^{1}\right)$, from the definition of $\lambda(u)$ in (4.2), one has $\lambda(u)=s_{i n}^{2}$. Therefore, from (4.1), we conclude $s_{2}^{+}(u)=\tilde{s}_{2}$ and then $\phi(u)=\mu_{2}\left(\tilde{s}_{2}\right)$ for all $u \geq \mu_{1}\left(s_{i n}^{1}\right)$, which proves part (a).

If $\tilde{s}_{2}=s_{2}^{\max }$, then $\lambda(u)=s_{i n}^{1}+s_{i n}^{2}-\tilde{s}_{1}^{*}(u) \geq s_{2}^{\max }$ for all $u \geq 0$. Therefore, $s_{2}^{+}(u)=s_{2}^{\max }=\tilde{s}_{2}$, and then $\phi(u)=\mu_{2}\left(\tilde{s}_{2}\right)$ for all $u \geq 0$, proving the second statement of part (b). For proving that $\phi$ is non-increasing, note that:

- the function $u \rightarrow \tilde{s}_{1}^{*}(u)$ defined in (4.3) is non-decreasing; hence, $u \rightarrow \lambda(u)$ is non-increasing, and therefore, $u \rightarrow s_{2}^{+}(u)=\min \left\{\lambda(u), s_{2}^{\max }\right\}$ is a non-increasing function;

- one has $s_{2}^{+}(u) \in\left[0, s_{2}^{\max }\right]$ for all $u \geq 0$, interval where function $\mu_{2}$ is increasing.

Therefore, $\phi(u)=\mu_{2}\left(s_{2}^{+}(u)\right)$ is a non-increasing function.

Now, part (c) is a direct consequence of the equality $\lambda(0)=s_{i n}^{1}+s_{i n}^{2}$, which ends the proof.

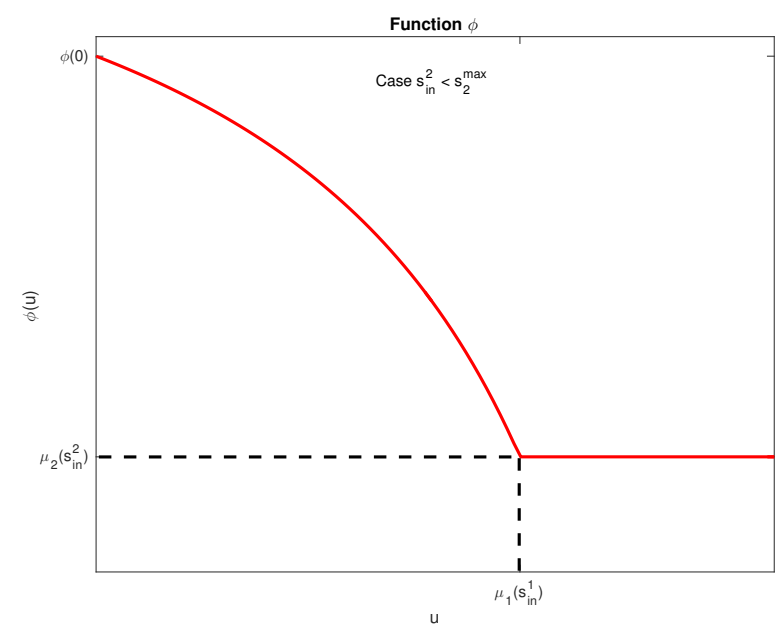

Figure 1: Plot of the function $\phi$ where the parameter values of functions $\tilde{\mu}_{1}$ and $\tilde{\mu}_{2}$ (and then of $\mu_{1}$ and $\mu_{2}$ ) are given in Table 2 in Section 5 (obtained from [4]) and $S_{i n}^{1}=5[\mathrm{~g} / \mathrm{l}]$ and $S_{i n}^{2}=10[\mathrm{mmol} / \mathrm{l}]$.

Since $I(u)=[0, \phi(u)]$, the previous result will imply an equivalent expression of the condition $u \in I(u)$, as the next corollary shows. 
Corollary 4.1. There exists a unique $u^{*}=u^{*}\left(s_{i n}^{1}, s_{i n}^{2}\right)>0$ such that $\phi\left(u^{*}\right)=u^{*}$ and

$$
u \in I(u) \Leftrightarrow u \in\left[0, u^{*}\right]
$$

We can be more specific for describing $u^{*}$ in the above corollary, as we establish in the next proposition.

Proposition 4.1. Condition $u \in I(u)$ is equivalent to requiring that $u$ belongs to a fixed interval, as follows:

- If $\mu_{1}\left(s_{i n}^{1}\right)<\mu_{2}\left(\tilde{s}_{2}\right)$, then

where $\tilde{s}_{2}$ is defined in (4.7).

$$
u \in I(u) \Leftrightarrow u \in\left[0, \mu_{2}\left(\tilde{s}_{2}\right)\right]
$$

- If $\mu_{1}\left(s_{i n}^{1}\right) \geq \mu_{2}\left(\tilde{s}_{2}\right)$, then

$$
u \in I(u) \Leftrightarrow u \in\left[0, u^{*}\right] \subset\left[0, \mu_{1}\left(s_{i n}^{1}\right)\right],
$$

where $u^{*} \in\left(0, \mu_{1}\left(s_{i n}^{1}\right)\right)$ is the unique positive solution of the equation

$$
u=\mu_{2}\left(\lambda_{-}(u)\right)
$$

with $\lambda_{-}(u)=\min \left\{s_{\text {in }}^{1}+s_{\text {in }}^{2}-s_{1}^{*}(u), s_{2}^{\max }\right\}$.

Proof. Let $u^{*}=u^{*}\left(s_{i n}^{1}, s_{i n}^{2}\right)$ be given by Corollary 4.1. If $\mu_{1}\left(s_{i n}^{1}\right)<\mu_{2}\left(\tilde{s}_{2}\right)=\phi\left(\mu_{1}\left(s_{i n}^{1}\right)\right)$ (the last equality is due to Lemma $4.1(\mathrm{i}))$, then one has $u^{*}>\mu_{1}\left(s_{i n}^{1}\right)$. Since $\phi(u)=\mu_{2}\left(\tilde{s}_{2}\right)$ for all $u \geq \mu_{1}\left(s_{i n}^{1}\right)$, we conclude

$$
u^{*}=\phi\left(u^{*}\right)=\mu_{2}\left(\tilde{s}_{2}\right),
$$

which together with (4.8) proves the first part of the proposition.

Similarly, if $\mu_{1}\left(s_{i n}^{1}\right) \geq \mu_{2}\left(\tilde{s}_{2}\right)=\phi\left(\mu_{1}\left(s_{i n}^{1}\right)\right)$, we obtain that $u^{*} \leq \mu_{1}\left(s_{i n}^{1}\right)$. Then,

$$
u \in I(u) \Leftrightarrow u \in\left[0, u^{*}\right] \subset\left[0, \mu_{1}\left(s_{i n}^{1}\right)\right] .
$$

Since $u^{*}=\phi\left(u^{*}\right)=\mu_{2}\left(s_{2}^{+}\left(u^{*}\right)\right), s_{2}^{+}\left(u^{*}\right)=\min \left\{\lambda\left(u^{*}\right), s_{2}^{\max }\right\} \quad($ see $(4.1))$ and $\lambda(u)=s_{\text {in }}^{1}+s_{\text {in }}^{2}-s_{1}^{*}(u)$ for $u \leq \mu_{1}\left(s_{i n}^{1}\right)$, we conclude $u^{*}=\mu_{2}\left(\lambda_{-}\left(u^{*}\right)\right)$.

With the characterization of the condition $u \in I(u)$ obtained previously, we can now rewrite the optimization problem (4.5). First, since $\tilde{s}_{1}^{*}(u)$ is piecewise (see (4.3)), function $g$ by (4.6) can be written

$$
g(u)= \begin{cases}g_{1}(u) & \text { if } u \in I(u) \text { and } u<\mu_{1}\left(s_{i n}^{1}\right), \\ g_{2}(u) & \text { if } u \in I(u) \text { and } u \geq \mu_{1}\left(s_{i n}^{1}\right),\end{cases}
$$

where

$$
\begin{array}{lll}
g_{1}(u):=u\left(s_{\text {in }}^{1}+s_{\text {in }}^{2}-s_{1}^{*}(u)-s_{2}^{*}(u)\right) & \text { for } u \in I(u) \text { and } u \in\left[0, \mu_{1}\left(s_{\text {in }}^{1}\right)\right) \\
g_{2}(u):=u\left(s_{\text {in }}^{2}-s_{2}^{*}(u)\right) & \text { for } u \in I(u), &
\end{array}
$$

together with the convention $s_{1}^{*}(0)=s_{2}^{*}(0)=0$ (indeed, one has $\left.\lim _{u \rightarrow 0^{+}} s_{1}^{*}(u)=\lim _{u \rightarrow 0^{+}} s_{2}^{*}(u)=0\right)$. Recall that $s_{1}^{*}(u)$ is the unique solution over $\left[0, s_{i n}^{1}\right]$ of the equation $\mu_{1}\left(s_{1}\right)=u$; that is, one has $s_{1}^{*}(u)=\mu_{1}^{-1}(u)$ (when $\left.u<\mu_{1}\left(s_{i n}^{1}\right)\right)$; and recall that $s_{2}^{*}(u)$ is the unique solution over $\left[0, s_{2}^{\text {max }}\right]$ of the equation $\mu_{2}\left(s_{2}\right)=u($ which exists when $u \in I(u))$.

Thanks to Proposition 4.1, functions $g, g_{1}$ and $g_{2}$ can be rewritten as follows:

1. If $\mu_{1}\left(s_{i n}^{1}\right)<\mu_{2}\left(\tilde{s}_{2}\right)$, then one has

$$
g(u)=\left\{\begin{array}{lr}
g_{1}(u) & \text { if } 0 \leq u<\mu_{1}\left(s_{i n}^{1}\right), \\
g_{2}(u) & \text { if } \quad \mu_{1}\left(s_{i n}^{1}\right) \leq u \leq \mu_{2}\left(\tilde{s}_{2}\right) .
\end{array}\right.
$$

together with

$$
\begin{aligned}
& g_{1}(u)=u\left(s_{i n}^{1}+s_{i n}^{2}-s_{1}^{*}(u)-s_{2}^{*}(u)\right) \quad \text { for } u \in\left[0, \mu_{1}\left(s_{i n}^{1}\right)\right] \text {, } \\
& g_{2}(u) \quad=u\left(s_{i n}^{2}-s_{2}^{*}(u)\right) \quad \text { for } u \in\left[0, \mu_{2}\left(\tilde{s}_{2}\right)\right] \text {, }
\end{aligned}
$$


2. If $\mu_{1}\left(s_{\text {in }}^{1}\right) \geq \mu_{2}\left(\tilde{s}_{2}\right)$, then one has

$$
g(u)=g_{1}(u)=u\left(s_{i n}^{1}+s_{i n}^{2}-s_{1}^{*}(u)-s_{2}^{*}(u)\right) \text { for all } u \in\left[0, u^{*}\right] \subset\left[0, \mu_{1}\left(s_{i n}^{1}\right)\right] .
$$

From (4.9) and (4.12), the optimization problem (4.5) presents the following structure.

Proposition 4.2. The maximization problem (4.5) is equivalent to one of the next two formulations:

- If $\mu_{1}\left(s_{i n}^{1}\right)<\mu_{2}\left(\tilde{s}_{2}\right)$, then problem (4.5) is equivalent to maximizing $g_{1}$ over $\left[0, \mu_{1}\left(s_{\text {in }}^{1}\right)\right]$ and to maximizing $g_{2}$ over $\left(\mu_{1}\left(s_{i n}^{1}\right), \mu_{2}\left(\tilde{s}_{2}\right)\right]$, and then the maximal value is taken between these two problems, that is:

$$
\max \left\{\max _{u \in\left[0, \mu_{1}\left(s_{i n}^{1}\right)\right]} g_{1}(u), \max _{u \in\left(\mu_{1}\left(s_{i n}^{1}\right), \mu_{2}\left(\tilde{s}_{2}\right)\right]} g_{2}(u)\right\} .
$$

- If $\mu_{1}\left(s_{i n}^{1}\right) \geq \mu_{2}\left(\tilde{s}_{2}\right)$, then problem (4.5) is equivalent to the optimization problem

$$
\max _{u \in\left[0, u^{*}\right]} g_{1}(u)
$$

where $u^{*}$ is the unique positive solution of the equation

$$
u=\mu_{2}\left(\lambda_{-}(u)\right),
$$

with $\lambda_{-}(u)=\min \left\{s_{i n}^{1}+s_{i n}^{2}-s_{1}^{*}(u), s_{2}^{\max }\right\}$. In this case, the maximal methane flow rate is achieved at a coexistence steady state $E_{4}^{*}(u)$ (see Remark 4.1).

Proof. It is a direct consequence from (4.9) and (4.12).

From the above proposition, when $\mu_{1}\left(s_{i n}^{1}\right) \geq \mu_{2}\left(\tilde{s}_{2}\right)$, the maximal methane flow rate at equilibrium is achieved at a steady state of coexistence $\left(i . e ., E_{4}^{*}(u)\right.$ ). This result is in line with other studies in the literature (see $[11,12])$. A plot of the mapping $g_{1}$ in this case can be found in Figure 2 (left). Nevertheless, if $\mu_{1}\left(s_{\text {in }}^{1}\right)<\mu_{2}\left(\tilde{s}_{2}\right)$, according to the first part of Proposition 4.2, the maximal methane flow rate at equilibrium could be achieved at some value $u \geq \mu_{1}\left(s_{i n}^{1}\right)$, that is, at a steady state of type $E_{2}^{*}(u)$ where the acidogenic microorganism is washed out (see Remark 4.1). This situation will occur when

$$
\max _{u \in\left[0, \mu_{1}\left(s_{i n}^{1}\right)\right]} g_{1}(u)<\max _{u \in\left[\mu_{1}\left(s_{i n}^{1}\right), \mu_{2}\left(\tilde{s}_{2}\right)\right]} g_{2}(u) .
$$

In order to give sufficient conditions ensuring that this case may happen, let us now give properties of the functions $g_{1}$ and $g_{2}$ (some of them illustrated in Figure 2) defining the optimization problem when $\mu_{1}\left(s_{\text {in }}^{1}\right)<$ $\mu_{2}\left(\tilde{s}_{2}\right)$.

Proposition 4.3. Suppose that $\mu_{1}\left(s_{\text {in }}^{1}\right)<\mu_{2}\left(\tilde{s}_{2}\right)$. Then, the functions $g, g_{1}$, and $g_{2}$ defined by (4.9), (4.10), and (4.11) satisfy the following properties:

(a) the function $g$ is continuous over $\left[0, \mu_{2}\left(\tilde{s}_{2}\right)\right]$;

(b) one has $g_{2}(u)<g_{1}(u)$ for all $u \in\left(0, \mu_{1}\left(s_{i n}^{1}\right)\right)$;

(c) the functions $g_{1}$ and $g_{2}$ are concave and differentiable over $\left(0, \mu_{1}\left(s_{i n}^{1}\right)\right)$ and $\left(0, \mu_{2}\left(\tilde{s}_{2}\right)\right)$, respectively. In addition, one has

$$
\begin{aligned}
& g_{2}^{\prime}(u)=s_{i n}^{2}-s_{2}^{*}(u)-\frac{u}{\mu_{2}^{\prime}\left(s_{2}^{*}(u)\right)}=s_{i n}^{2}-s_{2}^{*}(u)-\frac{\mu_{2}\left(s_{2}^{*}(u)\right)}{\mu_{2}^{\prime}\left(s_{2}^{*}(u)\right)} \quad \text { for } u \in\left(0, \mu_{2}\left(\tilde{s}_{2}\right)\right), \\
& g_{1}^{\prime}(u)=g_{2}^{\prime}(u)+s_{i n}^{1}-s_{1}^{*}(u)-\frac{u}{\mu_{1}^{\prime}\left(s_{1}^{*}(u)\right)}=g_{2}^{\prime}(u)+s_{i n}^{1}-s_{1}^{*}(u)-\frac{\mu_{1}\left(s_{1}^{*}(u)\right)}{\mu_{1}^{\prime}\left(s_{1}^{*}(u)\right)}
\end{aligned}
$$

for $u \in\left(0, \mu_{1}\left(s_{i n}^{1}\right)\right)$; 

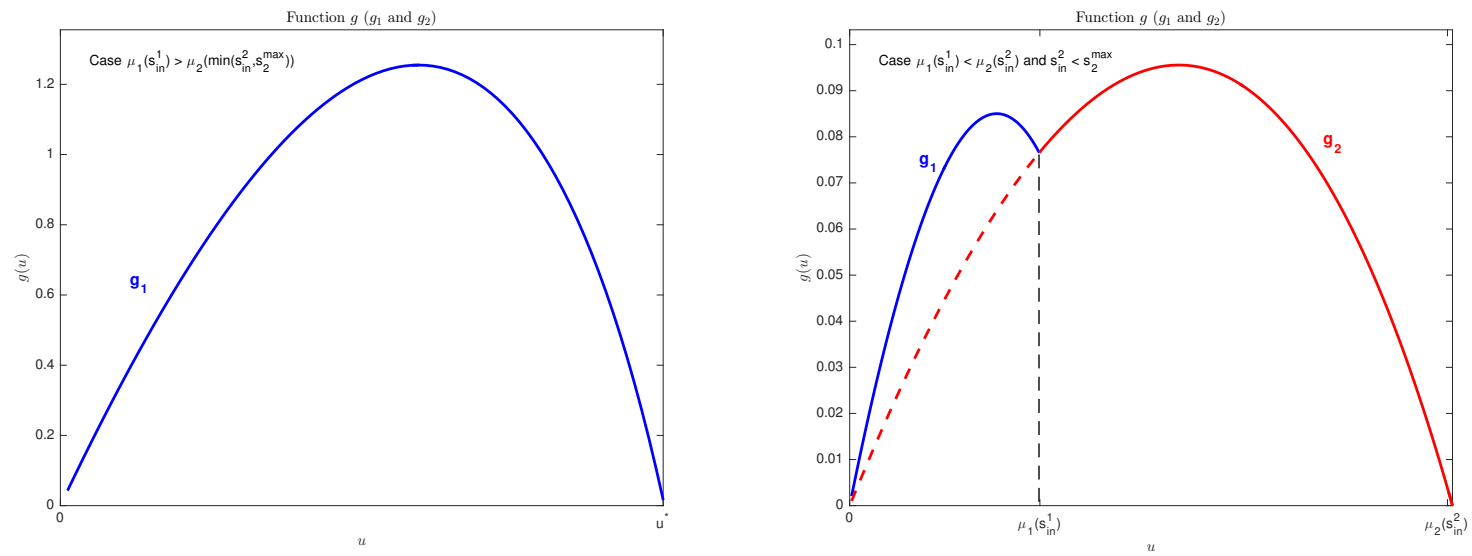

Figure 2: (Left): Plot of $g_{1}$ over $\left[0, u^{*}\right]$ in the case where $\mu_{1}\left(s_{i n}^{1}\right) \geq \mu_{2}\left(\tilde{s}_{2}\right)$ and $S_{\text {in }}^{1}=5[\mathrm{~g} / \mathrm{l}], S_{\text {in }}^{2}=10[\mathrm{mmol} / \mathrm{l}]$ (all other parameters are taken from Table 2 in Section 5). (Right): Plot of $g$ over $\left[0, \mu_{2}\left(\tilde{s}_{2}\right)\right]$ in the case where $\mu_{1}\left(s_{i n}^{1}\right)<\mu_{2}\left(\tilde{s}_{2}\right)$ and $S_{i n}^{1}=1.5[\mathrm{~g} / \mathrm{l}], S_{i n}^{2}=4[\mathrm{mmol} / \mathrm{l}], \bar{\mu}_{1}=0.4$ (all other parameters are taken from Table $2)$.

(d) one has $\lim _{u \rightarrow 0^{+}} g^{\prime}(u)=\lim _{u \rightarrow 0^{+}} g_{1}^{\prime}(u)>0 \quad$ and

$$
\lim _{u \rightarrow \mu_{2}\left(\tilde{s}_{2}\right)^{-}} g^{\prime}(u)=\lim _{u \rightarrow \mu_{2}\left(\tilde{s}_{2}\right)^{-}} g_{2}^{\prime}(u)= \begin{cases}-\infty & \text { if } \tilde{s}_{2}=s_{2}^{\max }, \\ -\frac{\mu_{2}\left(s_{i n}^{2}\right)}{\mu_{2}^{\prime}\left(s_{i n}^{2}\right)} & \text { if } \tilde{s}_{2}=s_{i n}^{2} .\end{cases}
$$

(e) $\lim _{u \rightarrow \mu_{1}\left(s_{i n}^{1}\right)^{-}} g^{\prime}(u)=\lim _{u \rightarrow \mu_{1}\left(s_{i n}^{1}\right)^{-}} g_{1}^{\prime}(u)<g_{2}^{\prime}\left(\mu_{1}\left(s_{i n}^{1}\right)\right)$.

Proof. Let us prove (a) and (b). From the definition of $g_{1}$ and $g_{2}$ in (4.10), and (4.11), one has

$$
g_{1}(u)=g_{2}(u)+u\left(s_{i n}^{1}-s_{1}^{*}(u)\right) .
$$

Since $s_{i n}^{1}>s_{1}^{*}(u)$ for $u \in\left[0, \mu_{1}\left(s_{i n}^{1}\right)\right)$ and $s_{i n}^{1}=s_{1}^{*}(u)$ when $u=\mu_{1}\left(s_{i n}^{1}\right)$, from (4.15) we deduce part (b) and also part (a) because functions $s_{1}^{*}(u)=\mu_{1}^{-1}(u)$ and $s_{2}^{*}(u)=\min \left\{s \mid \mu_{2}(s)=u\right\}$ are two continuous functions over $\left[0, \mu_{1}\left(s_{i n}^{1}\right)\right]$ and $\left[0, \mu_{2}\left(\tilde{s}_{2}\right)\right]$, respectively.

To prove (c), observe first that one has $u=\mu_{j}\left(s_{j}^{*}(u)\right)$ (for $u$ where $s_{j}^{*}(u)$ is well defined), implying

$$
\left(s_{j}^{*}\right)^{\prime}(u)=\frac{1}{\mu_{j}^{\prime}\left(s_{j}^{*}(u)\right)} \quad j \in\{1,2\}
$$

Thus, we get (4.13) and (4.14). Note also that the mappings $u \mapsto s_{j}^{*}(u)$ for $j \in\{1,2\}$ are increasing in the intervals where they are defined (recall assumptions 2.1 and 2.2, and Remark 2.2).

Finally, since $s_{2}^{*}(u) \in\left[0, s_{2}^{\max }\right]$ and $\mu_{2}^{\prime}$ is decreasing on this interval (indeed, $\mu_{2}$ is concave over $\left[0, s_{2}^{\max }\right]$ ), we deduce that $g_{2}^{\prime}$ is decreasing, implying that $g_{2}$ is concave. Analogously (because $\mu_{1}$ is concave) one can show that $g_{1}$ is also concave, which proves (c).

In order to prove $(\mathrm{d})$ and $(\mathrm{e})$, we can check that one has:

(i) $\mu_{1}^{\prime}(0)>0$ and $\mu_{2}^{\prime}(0)>0$ (from assumptions 2.1 and 2.2, and Remark 2.2);

(ii) $\lim _{u \rightarrow \mu_{2}\left(\tilde{s}_{2}\right)^{-}} s_{2}^{*}(u)=\tilde{s}_{2}$ and then $\mu_{2}^{\prime}\left(s_{2}^{*}(u)\right) \rightarrow 0$ if $\tilde{s}_{2}=s_{2}^{\max }$ or $\mu_{2}^{\prime}\left(s_{2}^{*}(u)\right) \rightarrow \mu_{2}^{\prime}\left(s_{i n}^{2}\right)$ if $\tilde{s}_{2}=s_{i n}^{2}$;

(iii) $\lim _{u \rightarrow \mu_{1}\left(s_{i n}^{1}\right)^{-}} g_{1}^{\prime}(u)=g_{2}^{\prime}\left(\mu_{1}\left(s_{i n}^{1}\right)\right)-\frac{\mu_{1}\left(s_{i n}^{1}\right)}{\mu_{1}^{\prime}\left(s_{i n}^{1}\right)}$, therefore $\lim _{u \rightarrow \mu_{1}\left(s_{i n}^{1}\right)^{-}} g_{1}^{\prime}(u)<g_{2}^{\prime}\left(\mu_{1}\left(s_{i n}^{1}\right)\right)$. 
Property (iii) straightforwardly implies (e). Now, with properties (ii) and (iii) together with the equalities $s_{1}^{*}(0)=s_{2}^{*}(0)=0$ and the expressions of $g_{1}^{\prime}$ and $g_{2}^{\prime}$ given by part (c), we can prove $(\mathrm{d})$.

When $\mu_{1}\left(s_{i n}^{1}\right)<\mu_{2}\left(\tilde{s}_{2}\right)$, the maximal value for the methane flow rate at steady state can then be obtained either at an equilibrium point of type $E_{2}^{*}(u)$ (i.e., when the acidogenic microorganism is washed out) or at an equilibrium point of type $E_{4}^{*}(u)$ (i.e., when there is coexistence of the two species). In the next result, we provide sufficient conditions that allow us to determine which equilibrium provides the greatest value for the methane flow rate.

Proposition 4.4. Suppose that $\mu_{1}\left(s_{\text {in }}^{1}\right)<\mu_{2}\left(\tilde{s}_{2}\right)$.

(a) If $\lim _{u \rightarrow \mu_{1}\left(s_{i n}^{1}\right)^{-}} g_{1}^{\prime}(u) \geq 0$, then one has

$$
\max _{u \in\left[0, \mu_{1}\left(s_{i n}^{1}\right)\right]} g_{1}(u)<\max _{u \in\left[\mu_{1}\left(s_{i n}^{1}\right), \mu_{2}\left(\tilde{s}_{2}\right)\right]} g_{2}(u),
$$

and therefore, the maximal methane flow rate at equilibrium in problem (4.5) is achieved for some $u \in\left(\mu_{1}\left(s_{i n}^{1}\right), \mu_{2}\left(\tilde{s}_{2}\right)\right)$, with the corresponding steady state $E_{2}^{*}(u)$ (washout of the acidogenic microorganism).

(b) If $g_{2}^{\prime}\left(\mu_{1}\left(s_{i n}^{1}\right)\right) \leq 0$, then one has

$$
\max _{u \in\left[0, \mu_{1}\left(s_{i n}^{1}\right)\right]} g_{1}(u) \geq \max _{u \in\left[\mu_{1}\left(s_{i n}^{1}\right), \mu_{2}\left(\tilde{s}_{2}\right)\right]} g_{2}(u),
$$

implying that the maximal methane flow rate at equilibrium in problem (4.5) is achieved for some $u \in\left(0, \mu_{1}\left(s_{i n}^{1}\right)\right)$, with the corresponding steady state $E_{4}^{*}(u)$ (coexistence).

Proof. If $\lim _{u \rightarrow \mu_{1}\left(s_{i n}^{1}\right)^{-}} g_{1}^{\prime}(u) \geq 0$, then we obtain

$$
\max _{u \in\left[0, \mu_{1}\left(s_{i n}^{1}\right)\right]} g_{1}(u)=g_{1}\left(\mu_{1}\left(s_{i n}^{1}\right)\right)=g_{2}\left(\mu_{1}\left(s_{i n}^{1}\right)\right),
$$

due to the concavity of $g_{1}$ and continuity of $g$ (see parts (a) and (c) in Proposition 4.3). Since $g_{2}$ is also concave and we have

$$
0 \leq \lim _{u \rightarrow \mu_{1}\left(s_{i n}^{1}\right)^{-}} g_{1}^{\prime}(u)<g_{2}^{\prime}\left(\mu_{1}\left(s_{i n}^{1}\right)\right),
$$

due to part (e) in Proposition 4.3, we conclude (4.16).

Finally, if $g_{2}^{\prime}\left(\mu_{1}\left(s_{i n}^{1}\right)\right) \leq 0$, we then necessarily have

$$
\max _{u \in\left[0, \mu_{1}\left(s_{i n}^{1}\right)\right]} g_{1}(u)=g_{1}(\bar{u})>g_{2}\left(\mu_{1}\left(s_{i n}^{1}\right)\right),
$$

for some $\bar{u} \in\left(0, \mu_{1}\left(s_{i n}^{1}\right)\right)$ where $g_{1}^{\prime}(\bar{u})=0$, because $g_{1}$ is concave and $\lim _{u \rightarrow 0^{+}} g^{\prime}(u)=\lim _{u \rightarrow 0^{+}} g_{1}^{\prime}(u)>0($ part $(\mathrm{d})$ in Proposition 4.3). By the concavity of $g_{2}$, one has that $g_{2}^{\prime}$ is negative over $\left[\mu_{1}\left(s_{i n}^{1}\right), \mu_{2}\left(\tilde{s}_{2}\right)\right]$, and therefore, we obtain (4.17).

\section{Numerical examples}

As established in the previous section, given acidogenic and methanogenic populations, characterized by functions $\mu_{1}(\cdot)$ and $\mu_{2}(\cdot)$ and the corresponding input substrate concentrations $s_{i n}^{1}$ and $s_{i n}^{2}$, the steady state that maximizes the methane flow rate at equilibrium can be of two types. Either the optimal steady state for (4.5) corresponds to the coexistence of both populations (if the optimal constant $u$ is lower than $\mu_{1}\left(s_{\text {in }}^{1}\right)$ ), or it corresponds to the washout of the acidogenic population. In this section, considering the original variables and parameters of system (2.1) obtained from [4], we will depict in the plane $S_{i n}^{1}-S_{i n}^{2}$ the set of points for which either the coexistence steady state or the wash-out steady state is optimal.

First, let us summarize analytically the conditions on the input substrate concentrations $S_{i n}^{1}$ and $S_{\text {in }}^{2}$, considering the original variables and parameters of system (2.1) in order to have the mentioned situations: 
- If $\tilde{\mu}_{1}\left(S_{i n}^{1}\right) \geq \tilde{\mu}_{2}\left(\tilde{S}_{2}\right)$, where

$$
\tilde{S}_{2}:=\min \left\{S_{2}^{\max }, S_{i n}^{2}\right\}
$$

then the maximal biogas flow rate is attained in a coexistence steady state (see Proposition 4.2 and Table 1);

- If $\tilde{\mu}_{1}\left(S_{i n}^{1}\right)<\tilde{\mu}_{2}\left(\tilde{S}_{2}\right)$ and $g_{2}^{\prime}\left(\tilde{\mu}_{1}\left(S_{i n}^{1}\right)\right) \leq 0$; then, the maximal biogas flow rate is attained in a coexistence steady state (see propositions 4.2 and 4.4 , and Table 1 );

- If $\tilde{\mu}_{1}\left(S_{i n}^{1}\right)<\tilde{\mu}_{2}\left(\tilde{S}_{2}\right)$ and $\lim _{u \rightarrow \tilde{\mu}_{1}\left(S_{i n}^{1}\right)^{-}} g_{1}^{\prime}(u) \geq 0$; then, the maximal biogas flow rate is attained in a steady state where the acidogenic population is washed out (see propositions 4.2 and 4.4, and and Table 1).

In order to determine in the plane $S_{i n}^{1}-S_{i n}^{2}$ which case occurs, we proceed as follows. Using the expressions of $g_{1}^{\prime}$ and $g_{2}^{\prime}$ in part (c) of Proposition 4.3, we compute the curves corresponding to equalities in the above conditions:

$$
\begin{aligned}
& C_{1}:=\left\{\left(S_{i n}^{1}, S_{i n}^{2}\right) \in \mathbb{R}_{+} \times \mathbb{R}_{+} ; \tilde{\mu}_{1}\left(S_{i n}^{1}\right)=\tilde{\mu}_{2}\left(\tilde{S}_{2}\right)\right\} \\
& C_{2}:=\left\{\left(S_{i n}^{1}, S_{i n}^{2}\right) \in \mathbb{R}_{+} \times \mathbb{R}_{+} ; g_{2}^{\prime}\left(\tilde{\mu}_{1}\left(S_{i n}^{1}\right)\right)=0\right\}, \\
& C_{3}:=\left\{\left(S_{i n}^{1}, S_{i n}^{2}\right) \in \mathbb{R}_{+} \times \mathbb{R}_{+} ; \lim _{u \rightarrow \tilde{\mu}_{1}\left(S_{i n}^{1}\right)^{-}} g_{1}^{\prime}(u)=0\right\} .
\end{aligned}
$$

It is worth to mention that

$$
\begin{aligned}
& \left(S_{i n}^{1}, S_{i n}^{2}\right) \in C_{2} \quad \Leftrightarrow \quad S_{i n}^{2}=S_{2}^{*}\left(\tilde{\mu}_{1}\left(S_{i n}^{1}\right)\right)+\frac{\tilde{\mu}_{2}\left(S_{2}^{*}\left(\tilde{\mu}_{1}\left(S_{i n}^{1}\right)\right)\right)}{\tilde{\mu}_{2}^{\prime}\left(S_{2}^{*}\left(\tilde{\mu}_{1}\left(S_{i n}^{1}\right)\right)\right)} \\
& \left(S_{i n}^{1}, S_{i n}^{2}\right) \in C_{3} \quad \Leftrightarrow \quad S_{i n}^{2}=S_{2}^{*}\left(\tilde{\mu}_{1}\left(S_{i n}^{1}\right)\right)+\frac{k_{2}}{k_{1}} \frac{\tilde{\mu}_{1}\left(S_{i n}^{1}\right)}{\tilde{\mu}_{1}^{\prime}\left(S_{i n}^{1}\right)}+\frac{\tilde{\mu}_{2}\left(S_{2}^{*}\left(\tilde{\mu}_{1}\left(S_{i n}^{1}\right)\right)\right)}{\mu_{2}^{\prime}\left(S_{2}^{*}\left(\mu_{1}\left(S_{i n}^{1}\right)\right)\right)} .
\end{aligned}
$$

In the above expressions, $S_{2}^{*}(u)$ stands for the unique solution in $\left[0, S_{2}^{\max }\right]$ of the equation $\tilde{\mu}_{2}(S)=u$.

Remark 5.1. Note that if $\tilde{\mu}_{1}\left(S_{i n}^{1}\right) \geq \tilde{\mu}_{2}\left(S_{2}^{\max }\right)$, then $\tilde{\mu}_{1}\left(S_{i n}^{1}\right) \geq \tilde{\mu}_{2}\left(\tilde{S}_{2}\right)$ for all $S_{i n}^{2} \geq 0$, and therefore in this case, the optimal methane flow rate at equilibrium is attained for a coexistence steady state. For this reason, in the figure below, we restrict the plane $S_{i n}^{1}-S_{i n}^{2}$ to values $\left(S_{i n}^{1}, S_{i n}^{2}\right)$ such that $0 \leq S_{i n}^{1} \leq \tilde{\mu}_{1}^{-1}\left(\tilde{\mu}_{2}\left(S_{2}^{\text {max }}\right)\right)$. Supposing that $S_{i n}^{1} \leq \tilde{\mu}_{1}^{-1}\left(\tilde{\mu}_{2}\left(S_{2}^{\text {max }}\right)\right)$ ensures that the washout of the acidogenic population could be optimal.

Figure 3 has been obtained using the parameter values that can be found in [4]. This allows us to obtain the values of $\tilde{\mu}_{1}(\cdot)$ and $\tilde{\mu}_{2}(\cdot)$, Monod and Haldane, respectively (see Example 2.1 ), and also the values of the pseudostochiometric coefficients in (2.1) together with the expression of (2.2). All these parameter values are specified in Table 2 .

\begin{tabular}{|c|c|c|}
\hline Parameter & Value & Unit \\
\hline$\alpha$ & 0.5 & \\
\hline $\bar{\mu}_{1}$ & 1.2 & $\mathrm{~d}^{-1}$ \\
\hline$k_{A}$ & 7.1 & $\mathrm{~g} / \mathrm{l}$ \\
\hline $\bar{\mu}_{2}$ & 0.74 & $\mathrm{~d}^{-1}$ \\
\hline$k_{M}$ & 9.28 & $\mathrm{mmol} / \mathrm{l}$ \\
\hline$k_{I}$ & 256 & $\mathrm{mmol} / \mathrm{l}$ \\
\hline$k_{1}$ & 42.14 & \\
\hline$k_{2}$ & 116.5 & $\mathrm{mmol} / \mathrm{g}$ \\
\hline$k_{3}$ & 268 & $\mathrm{mmol} / \mathrm{g}$ \\
\hline $\bar{q}$ & 453 & $\mathrm{mmol} / \mathrm{g}$ \\
\hline
\end{tabular}

Table 2: Values of parameters involved in system (2.1) obtained from [4]. 


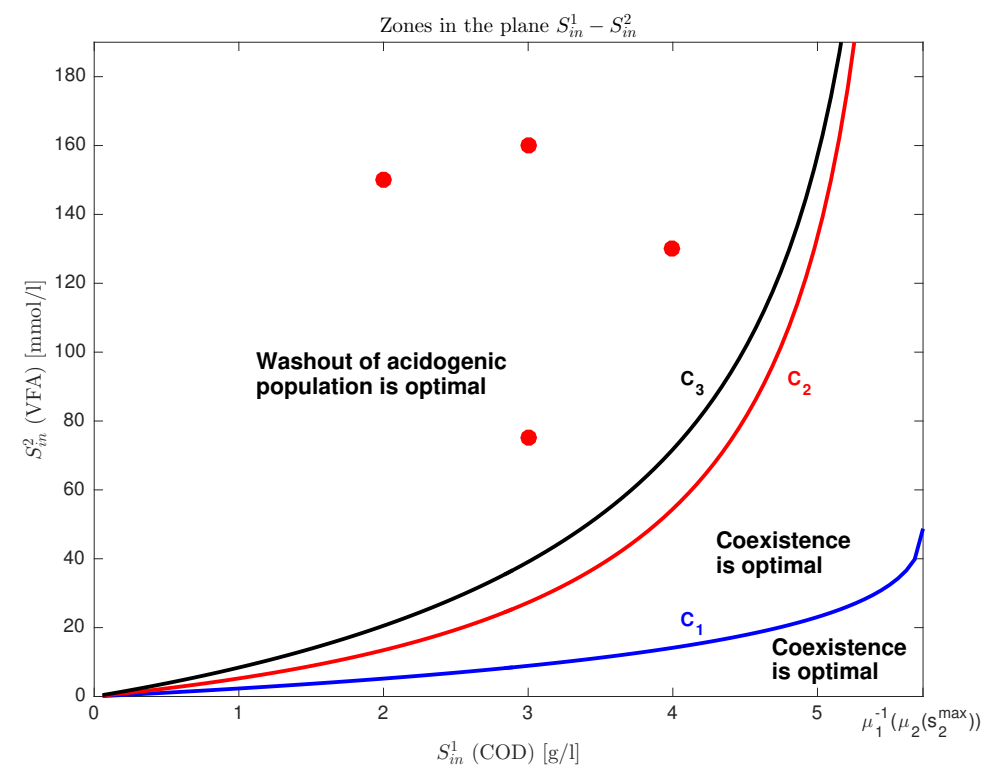

Figure 3: The three curves $C_{1}, C_{2}, C_{3}$ delimit in the plane $S_{i n}^{1}-S_{i n}^{2}$ three subsets where either coexistence or washout of acidogenic population is optimal for the maximization of biogas at steady state.

In Figure 3, we have depicted four points in the plane $S_{i n}^{1}-S_{i n}^{2}$, according to concentrations of $S_{i n}^{1}$ and $S_{i n}^{2}$ belonging to the range published in [16] for these quantities. Since these points belong to the subset where the wash-out of the acidogenic population is optimal, we present in Table 3 the optimal methane gas flow rate given by (2.2) together with the gain with respect to the other strategy corresponding to a coexistence steady state.

\begin{tabular}{|c|c|c|c|c|c|}
\hline$S_{\text {in }}^{1}(\mathbf{C O D})[\mathrm{g} / \mathbf{l}]$ & $S_{\text {in }}^{2}$ (VFA) $[\mathbf{m m o l} / \mathbf{l}]$ & $D_{\text {opt }}\left[\mathbf{d}^{-1}\right]$ & $S_{2}^{*}\left(\alpha D_{\text {opt }}\right)[\mathbf{m m o l} / \mathbf{l}]$ & $q_{M}[\mathbf{m m o l} / \mathbf{l d}]$ & $\mathbf{G a i n}$ \\
\hline 2 & 150 & 1.00 & 24.34 & 212.93 & $64.95 \%$ \\
\hline 3 & 160 & 1.01 & 25.05 & 229.92 & $26.31 \%$ \\
\hline 3 & 75 & 0.92 & 17.11 & 89.99 & $13.02 \%$ \\
\hline 4 & 130 & 0.99 & 22.79 & 179.25 & $5.84 \%$ \\
\hline
\end{tabular}

Table 3: Gain of methane flow rate at steady state when considering the wash-out of the acidogenic population in place of coexistence equilibrium.

\section{Conclusions}

In the last years, simplified models have been developed for monitoring and controlling anaerobic digestion processes in order to optimize the functioning of them. The analysis of simple models such as the twostage anaerobic digestion model proposed in [4] have been carried out by several authors (see, for instance, $[11,12,13,3]$ and the references therein). As noted by these authors, steady states of the model introduced in [4] can be as follows: (i) the extinction (washout) of one or two of the involved microorganisms; (ii) the coexistence of microorganisms. The coexistence steady states are referred to as nominal operating points, and they are usually viewed as the desired operating conditions. From a practical point of view, an interesting problem is to obtain an efficient management policy of such bioprocesses in order to maximize the biogas production over a given period. This policy can be to find an equilibrium point maximizing the biogas production at the steady state. Intuitively, the optimal steady states should be nominal operating points (coexistence 
of microorganims), as assumed in [11, 12]. Nevertheless, as we have shown in this paper, under some simple assumptions related to input substrate concentrations and microorganism growth functions, the optimal steady state can be the extinction of one of the species, a phenomena that can be observed numerically in the three-stage model introduced in [15] and in the four-stage model proposed in [5]. This non-intuitive result can be interesting from a practical point of view if the goal is to maximize biogas production. In particular, if in the effluent to be treated the total concentration $S_{i n}^{2}$ of VFA is too high with respect to the organic substrate concentration $S_{i n}^{1}$ (characterized by its COD), in the sense that $\tilde{\mu}_{1}\left(S_{i n}^{1}\right)<\tilde{\mu}_{2}\left(S_{2}^{\max }\right)$, it could be optimal to wash out the acidogenic population. We have noted that this can happen when taking values from the literature; specifically, we considered parameters of acidogenic and methanogenic populations from [4] and concentrations of organic substrate and VFA of effluents from the wine industry published in [16].

It is worthwhile to point out that in general, the VFA concentration in the effluent to be treated is not very high, and therefore, the optimal methane flow rate is attained in the coexistence of microorganisms. Nevertheless, due to storage or transport of the water to be treated, the VFA concentration could increase considerably (as shown in [16]), in these cases it being better to wash out the acidogenic microorganisms in order to maximize methane production. Therefore, we believe that the analytic characterization of regions in the plane $S_{i n}^{1}-S_{i n}^{2}$, where the coexistence or the washout of acidogenic population is optimal, could help in the design of an anaerobic digestion process, for instance, considering two interconnected bioreactors where the objective is to maximize methane production. In this setting, one can consider a first tank with only methanogenic microorganisms and receiving an effluent to be treated; this tank is connected with a second tank where the two populations coexist. This second bioreactor receives the flow treated from the first tank and can also receive the effluent to be treated without passing by the first tank. Thus, our analytical results can be used in order to determine if the effluent to be treated (taking into account its concentrations of $S_{\text {in }}^{1}$ and $S_{i n}^{2}$ ) should pass by the first bioreactor (if $\tilde{\mu}_{1}\left(S_{i n}^{1}\right)<\tilde{\mu}_{2}\left(S_{2}^{\max }\right)$ ) or directly by the second bioreactor in order to maximize the methane flow rate.

\section{Acknowledgments}

This paper has benefited considerably from advice and comments by Andrés Donoso (INRIA Chile) and Jérôme Harmand (INRA France), although they should not be held responsible for any mistake. The authors are very grateful to them. The first author would like to thank INRA Montpellier and the UMR MISTEA for providing a half year delegation during the academic year 2017-2018. This research benefited from the support of FONDECYT grant (Chile) N 1160567 and Proyecto Redes 150011 (Chile). The second author was also partially supported by Basal Project CMM Universidad de Chile.

\section{References}

[1] D. J. Batstone, J. Keller, I. Angelidaki, S. V. Kalyuzhnyi, S. G. Pavlostathis, A. Rozzi, W. Sanders, H. Siegrist, AND V. A. Vavilin, The iwa anaerobic digestion model no 1 (adm1), Water Science and Technology, 45 (2002), pp. 65-73.

[2] T. Bayen, A. Rapaport, and M. Sebbah, Minimal time control of the two tanks gradostat model under a cascade inputs constraint, SIAM J. Control Optim., 52 (2014), pp. 2568-2594.

[3] B. Benyahia, T. Sari, B. Cherki, And J. Harmand, Bifurcation and stability analysis of a two step model for monitoring anaerobic digestion processes, Journal of Process Control, 22 (2012), pp. 1008 1019.

[4] O. Bernard, Z. Hadj-Sadok, D. Dochain, A. Genovesi, and J.-P. Steyer, Dynamical model development and parameter identification for an anaerobic wastewater treatment process, Biotechnology and bioengineering, 75 (2001), pp. 424-438.

[5] Y. Daoud, N. Abdellatif, and J. Harmand, Modèles mathématiques de digestion anaérobie: effet de l'hydrolyse sur la production du biogaz, (2017). https://hal.archives-ouvertes.fr/hal-01562353/ document. 
[6] A. Donoso-Bravo, J. Mailier, C. Martin, J. Rodríguez, C. A. Aceves-Lara, and A. VANDE Wouwer, Model selection, identification and validation in anaerobic digestion: a review, Water research, 45 (2011), pp. 5347-5364.

[7] A. Haddon, J. Harmand, H. Ramírez, and A. Rapaport, Guaranteed value strategy for the optimal control of biogas production in continuous bio-reactors, in IFAC Word Congress 2017, July 9-14, 2017.

[8] A. Kelessidis And A. S. Stasinakis, Comparative study of the methods used for treatment and final disposal of sewage sludge in european countries, Waste management, 32 (2012), pp. 1186-1195.

[9] M. Kim, Y.-H. Ahn, And R. SpeECE, Comparative process stability and efficiency of anaerobic digestion; mesophilic vs. thermophilic, Water research, 36 (2002), pp. 4369-4385.

[10] A. Novick ANd L. SzILARd, Experiments with the chemostat on spontaneous mutations of bacteria, Proceedings of the National Academy of Sciences, 36 (1950), pp. 708-719.

[11] M. Sbarciog, M. Loccufier, And A. Vande Wouwer, On the optimization of biogas production in anaerobic digestion systems, IFAC Proceedings Volumes, 44 (2011), pp. 7150-7155.

[12] M. Sbarciog, M. Loccufier, and A. Vande Wouwer, An optimizing start-up strategy for a biomethanator, Bioprocess and biosystems engineering, 35 (2012), pp. 565-578.

[13] M. Sbarciog, J. A. Moreno, and A. Vande Wouwer, A biogas-based switching control policy for anaerobic digestion systems1, IFAC Proceedings Volumes, 45 (2012), pp. 603-608.

[14] H. L. Smith And P. Waltman, The theory of the chemostat, vol. 13 of Cambridge Studies in Mathematical Biology, Cambridge University Press, Cambridge, 1995. Dynamics of microbial competition.

[15] M. Weedermann, G. S. Wolkowicz, and J. Sasara, Optimal biogas production in a model for anaerobic digestion, Nonlinear Dynamics, 81 (2015), pp. 1097-1112.

[16] P. J. Welz, G. Holtman, R. Haldenwang, and M. Le Roes-Hill, Characterisation of winery wastewater from continuous flow settling basins and waste stabilisation ponds over the course of 1 year: implications for biological wastewater treatment and land application, Water Science and Technology, 74 (2016), pp. 2036-2050. 